\title{
COMPOSIÇÃO MINERAL, QUALIDADE E DEGENERESCÊNCIA DE POLPA DE MAÇÃS 'FUJI' EM DIFERENTES PORTA-ENXERTOS DURANTE ARMAZENAMENTO EM ATMOSFERA CONTROLADA ${ }^{1}$

\author{
THAIS ROSELI CORRÊA², CRISTIANO ANDRÉ STEFFENS ${ }^{3}$, \\ CASSANDRO VIDAL TALAMINI DO AMARANTE ${ }^{4}$, HÉLIO TANAKA ${ }^{5}$, \\ MAYARA CRISTIANA STANGER $^{2}$, AURI BRACKAMNN ${ }^{6}$, PAULO ROBERTO ERNANI ${ }^{7}$
}

RESUMO - O objetivo deste trabalho foi avaliar os efeitos de três porta-enxertos sobre a composição mineral em maçãs 'Fuji', bem como a qualidade e incidência de degenerescência de polpa dos frutos, armazenados em duas condições de atmosfera controlada (AC). Frutos provenientes de árvores com três porta-enxertos (MM-106, Marubakaido e Marubakaido com filtro M-9) provenientes de São Joaquim - SC foram armazenados por oito meses em duas condições de $\mathrm{AC}\left(1,2 \mathrm{kPa} \mathrm{O}_{2}+<0,5 \mathrm{kPa} \mathrm{CO}\right.$ e $1,2 \mathrm{kPa} \mathrm{O}_{2}+$ $2,0 \mathrm{kPa} \mathrm{CO}$ ). Sete dias após a retirada das câmaras, determinaram-se: taxa de produção de etileno, cor da epiderme $\left(h^{\circ}\right)$, força para a penetração da polpa, acidez titulável, sólidos solúveis, incidência e severidade de degenerescência de polpa e composição mineral $(\mathrm{Ca}, \mathrm{Mg}$ e $\mathrm{K})$ na polpa dos frutos. Entre condições de AC, os frutos armazenados com 2,0 $\mathrm{kPa}$ de $\mathrm{CO}_{2}$ apresentaram um retardo no amadurecimento se comparados aos frutos armazenados nas condições de $<0,5 \mathrm{kPa}$ de $\mathrm{CO}_{2}$. Quanto aos diferentes porta-enxertos, na condição de armazenamento com 2,0 kPa CO , os frutos de plantas com Marubakaido e Marubakaido com filtro-M9 apresentaram maior incidência de degenerescência de polpa se comparados aos frutos de plantas com MM106, que apresentaram maiores teores de Ca e menores teores de $\mathrm{K}$, e menor relação $\mathrm{K} / \mathrm{Ca}$. A qualidade de maçãs 'Fuji' é influenciada principalmente pela condição de armazenamento em AC e muito pouco pelo tipo de porta-enxerto. Plantas enxertadas com MM-106 proporcionam frutos com menor predisposição à degenerescência de polpa, que por sua vez está relacionada à composição mineral do fruto.

Termos para indexação: Malus domestica, pré-colheita, nutrição, armazenamento, injúria de $\mathrm{CO}_{2}$, distúrbio fisiológico.

\section{MINERAL COMPOSITION, QUALITY AND INTERNAL BREAKDOWN OF 'FUJI' APPLES IN DIFFERENT ROOTSTOCKS DURING CONTROLLED ATMOSPHERE STORAGE}

\begin{abstract}
This study was carried out to evaluate the effects of three rootstocks on mineral composition of 'Fuji' apples, as well as on their, quality and incidence of internal breakdown in two controlled atmosphere (CA) conditions. Fruits harvested from trees with different rootstocks (MM-106, Marubakaido and Marubakaido with M-9 interstock) were stored for eight months in two CA conditions $\left(1.2 \mathrm{kPa}\right.$ of $\mathrm{O}_{2}$ $+<0.5 \mathrm{kPa}$ of $\mathrm{CO}_{2}$ and $1.2 \mathrm{kPa}$ of $\mathrm{O}_{2}+2.0 \mathrm{kPa}$ of $\mathrm{CO}_{2}$ ). Seven days after removall from CA storage, fruits were assessed for ethylene production rate, skin color $\left(h^{\circ}\right)$, flesh penetration force, titratable acidity, soluble solids content, incidence and severity of internal breakdown and mineral composition $(\mathrm{Ca}, \mathrm{Mg}$ and $\mathrm{K})$ in the fruit pulp. Among the CA conditions, fruits stored in $2 \mathrm{kPa} \mathrm{CO}_{2}$ showed delayed in the ripening compared to fruits stored in $<0.5 \mathrm{kPa} \mathrm{CO}$. When comparing rootstocks, fruits harvested from trees on Marubakaido and Marubakaido with M-9 interstock and then stored in $2.0 \mathrm{kPa}$ of $\mathrm{CO}_{2}$ showed higher incidence of internal breakdown than fruits harvested from trees on MM-106 rootstock. Fruits harvested from trees on MM-106 rootstock had higher $\mathrm{Ca}$ and lower $\mathrm{K}$ contents, and lower $\mathrm{K} / \mathrm{Ca}$ ration, than fruits harvested from trees on Marubakaido and Marubakaido with M-9 interstock. Fruit quality of 'Fuji' apples is more affected by the CA storage condition than by rootstock of the trees. Fruits from trees on MM-106 rootstock had lower predisposition to internal breakdown, which in turn is related to the mineral composition of fruit
\end{abstract}

Index terms: Malus domestica, preharvest, nutrition, storage, $\mathrm{CO}_{2}$ injury, physiological disorder.

${ }_{1}^{1}$ (Trabalho 190-11). Recebido em: 04-07-2011. Aceito para publicação em: 08-12-2011.

${ }^{2}$ Acadêmicas do Curso de Mestrado em Produção Vegetal, Centro de Ciências Agroveterinárias (CAV), Universidade do Estado de Santa Catarina (UDESC). Av. Luiz de Camões, 2090, CEP 88520-000, Lages-SC. E-mails: thaisroselicorrea@hotmail.com (autor para correspondência); mayara.stanger@hotmail.com 


\section{INTRODUÇÃO}

_ Maçãs ‘Fuji' apresentam bom potencial de armazenamento em condições de atmosfera controlada (AC), tendo sua qualidade mantida por um período prolongado. Porém, a degenerescência de polpa é um dos maiores problemas verificados no seu armazenamento (BRACKMANN et al., 2002a). Esse distúrbio fisiológico está associado principalmente ao acúmulo do $\mathrm{CO}_{2}$ no ambiente de armazenamento. Os frutos afetados pelo alto $\mathrm{CO}_{2}$ (acima de $1 \mathrm{kPa}$ ) apresentam aspecto externo normal; contudo, ao serem cortados ao meio, visualizam-se regiões escurecidas da polpa que comprometem a qualidade e causam consideráveis perdas pós-colheita (CORRÊA et al., 2010).

Para evitar o surgimento da degenerescência de polpa em maçãs 'Fuji', no armazenamento em $\mathrm{AC}$, é recomendado o uso de pressões parciais de $\mathrm{CO}_{2}$ abaixo de $0,5 \mathrm{kPa}$ (BRACKMANN et al., 2002a). No entanto, quando os frutos são armazenados nestas condições por períodos prolongados (oito meses), a qualidade pode estar comprometida. Steffens e Brackmann (2007) verificaram que o armazenamento de maçãs 'Fuji' com $2 \mathrm{kPa}$ de $\mathrm{CO}_{2}$ não induziu o desenvolvimento de degenerescência da polpa e manteve a qualidade. Isto demonstra que, em determinados anos de produção, alguns fatores interferem na predisposição dos frutos à ocorrência de degenerescência da polpa, em função da sua sensibilidade ao $\mathrm{CO}_{2}$ durante o armazenamento em AC.

Dentre os fatores relacionados com a presença deste distúrbio fisiológico, tem-se a temperatura do ar durante a fase de crescimento dos frutos (CORRÊA et al., 2010), a presença de pingo-de-mel no momento da colheita (BRACKMANN et al., 2002) e a composição mineral dos frutos (IUCHI; IUCHI, 2001; CANTILLANO; GIRARDI, 2004).

Como componente mineral relacionado com a degenerescência de polpa, o cálcio pode ser o principal, pois este nutriente está envolvido com a estrutura, funcionalidade, estabilidade celular e degradação da lamela média após o armazenamento dos frutos (JAMES; JOBLIN, 2009). Todavia, mesmo em pomares cujo solo esteja bem suprido em $\mathrm{Ca}$ $\left(11 \mathrm{cmolc} / \mathrm{dm}^{3}\right)$, a baixa concentração deste nutriente nos frutos resulta no aparecimento de distúrbios fisiológicos (SAURE, 2005; AMARANTE et al., 2010 ).

A composição mineral e a qualidade de frutos podem ser influenciadas pelo comportamento do porta-enxerto, pois ele interfere na absorção de água e nutrientes (RATO et al., 2008; MARTÍNEZBALLESTA et al., 2010). Os porta-enxertos anões são capazes de direcionar mais nutrientes aos frutos devido à menor competição fornecida pela parte vegetativa da planta. Por outro lado, porta-enxertos vigorosos podem influenciar negativamente essa competição por nutrientes (REMORINI et al., 2008). Para Saure (2005), há uma competição por nutrientes entre folhas jovens e frutos, principalmente pelo $\mathrm{Ca}$, e, juntamente com uma capacidade insuficiente da planta em translocar esse nutriente, há também uma concorrência entre frutos de baixa transpiração e frutos de crescimento vigoroso. Desta forma, o portaenxerto pode exercer uma importante influência na ocorrência de distúrbios fisiológicos que surgem no armazenamento, como a degenerescência de polpa. Se este distúrbio estiver relacionado com o teor de nutrientes na polpa, o porta-enxerto pode interferir no aporte de nutrientes da planta e na composição mineral do fruto.

O objetivo deste trabalho foi avaliar os efeitos de três porta-enxertos sobre a composição mineral de frutos, a manutenção da qualidade e a incidência de degenerescência de polpa em maçãs 'Fuji', em combinação com duas condições de AC.

\section{MATERIAL E MÉTODOS}

O experimento foi conduzido no ano agrícola de 2008/2009, com maçãs 'Fuji' provenientes de árvores com três porta-enxertos (MM-106, Marubakaido e Marubakaido com filtro M-9) de um pomar comercial do município de São Joaquim-SC (situado a $28^{\circ} 11^{\prime} 19,66^{\prime}$ ' S de latitude, 49 59' 42,60' W de longitude e $1.219 \mathrm{~m}$ de altitude). Macieiras com porta-enxerto MM-106 apresentavam 31 anos de idade e espaçamento de 2,7 m (na linha) x 6,0 $\mathrm{m}$ (entre linhas). No porta-enxerto Marubakaido, as plantas apresentavam 21 anos de idade e espaçamento de $4 \mathrm{~m}$ x 6,0m. As plantas de porta-enxerto Marubakaido com filtro-M9 (com comprimento de $20 \mathrm{~cm}$ ) apresentavam 18 anos de idade e espaçamento de $1,5 \mathrm{~m} \mathrm{x}$ 5,0 m. Em todos os porta-enxertos, as plantas foram conduzidas sob líder central, As características do solo do pomar são: Neossolo Litólico, $\mathrm{pH}$ em água de 6,3; 22,3 $\mathrm{mg} \mathrm{dm}^{-3}$ de P; 3,9 $\mathrm{mmol}_{\mathrm{c}} \mathrm{dm}^{-3}$ de K; 91 $\mathrm{mmol}_{\mathrm{c}} \mathrm{dm}^{-3} \mathrm{de} \mathrm{Ca} ; 48 \mathrm{mmol}_{\mathrm{c}} \mathrm{dm}^{-3} \mathrm{de} \mathrm{Mg} ; 63 \mathrm{~g} \mathrm{dm}^{-3}$ de matéria orgânica e $230 \mathrm{~g} \mathrm{dm}^{-3}$ de argila.

Os frutos foram colhidos manualmente, colocados em sacolas de colheita e, após, acondicionados em bins de madeira. Destes frutos colocados em bins, foram aleatoriamente retirados aqueles que constituíram as amostras experimentais, descartando aqueles que apresentavam lesões, defeitos, ferimentos ou dano mecânico e, em seguida, efetuou-se a homo- 
geneização das unidades experimentais.

O delineamento experimental adotado foi o inteiramente casualizado, segundo um esquema fatorial completo $2 \times 3$ (duas condições de armazenamento e três porta-enxertos), com cada unidade experimental constituída por 40 frutos.

As condições para o armazenamento dos frutos em atmosfera controlada (AC) foram $1,2 \mathrm{kPa}$ de $\mathrm{O}_{2}+<0,5 \mathrm{kPa}$ de $\mathrm{CO}_{2}$ e $1,2 \mathrm{kPa}$ de $\mathrm{O}_{2}+2,0 \mathrm{kPa}$ de $\mathrm{CO}_{2}$, a $0,5 \pm 0,1^{\circ} \mathrm{C}$ e umidade relativa de $97 \%$. Tendo em vista que em determinados anos a incidência de degenerescência da polpa não ocorre ou ocorre em baixa frequência, utilizou-se no experimento a condição de $\mathrm{AC}$ com $2 \mathrm{kPa}$ de $\mathrm{CO}_{2}$ para estimular a manifestação do distúrbio nos frutos mais sensíveis, visto que a cultivar Fuji, pela sua sensibilidade ao alto $\mathrm{CO}_{2}$, manifesta mais sintomas de degenerescência nesta condição de armazenamento.

Os frutos de todos os tratamentos foram armazenados em microcâmaras experimentais com capacidade de $250 \mathrm{~L}$. As pressões parciais de $\mathrm{O}_{2}$ foram obtidas mediante sua diluição no ambiente de armazenamento com injeção de $\mathrm{N}_{2}$, proveniente de um gerador de nitrogênio, que utiliza o princípio "Pressure Swing Adsorption" (PSA). A pressão parcial de $\mathrm{CO}_{2}$ referente ao tratamento com alto $\mathrm{CO}_{2}(2,0 \mathrm{kPa})$ foi obtida mediante a injeção deste gás, proveniente de cilindro de alta pressão. A manutenção das pressões parciais desejadas dos gases, nas duas condições de armazenamento, que variavam em função da respiração dos frutos, foi realizada diariamente, com o uso de equipamento automático para controle de gases (Kronenberger/Climasul). Quando os níveis do $\mathrm{CO}_{2}$ e $\mathrm{O}_{2}$ não estavam adequados, o equipamento procedia à correção das pressões parciais até os níveis preestabelecidos nos tratamentos. $\mathrm{O} \mathrm{O}_{2}$ consumido pela respiração foi reposto por meio da injeção de ar atmosférico nas minicâmaras, e o $\mathrm{CO}_{2}$ em excesso (no tratamento com 2,0 kPa) foi absorvido por uma solução de hidróxido de potássio (40\%), através da qual foi circulado o ar do ambiente de armazenamento. No tratamento com baixo $\mathrm{CO}_{2}$, a pressão parcial $<0,5 \mathrm{kPa}$ foi mantida através da colocação de cal hidratada no interior das minicâmaras, para a contínua eliminação do $\mathrm{CO}_{2}$ no ambiente de armazenamento.

Os frutos foram analisados no momento da colheita, aos oito meses de armazenamento e após sete dias de exposição à temperatura ambiente $\left(20^{\circ} \mathrm{C}\right)$, simulando assim o período de comercialização. As variáveis analisadas foram taxa de produção de etileno, cor da epiderme, força para a penetração da polpa, acidez titulável (AT), teores de sólidos solúveis (SS), conforme descrito por Corrêa et al. (2010), incidência e severidade de degenerescência de polpa, e teores de $\mathrm{Ca}, \mathrm{Mg}$ e K.

A incidência de degenerescência da polpa (\%) foi avaliada conforme descrito por Corrêa et al. (2010). Sequencialmente, os frutos foram agrupados de acordo com a seguinte escala de severidade: $1=$ sem incidência de degenerescência de polpa; $2=$ incidência de degenerescência leve, com até 10\% da polpa afetada; 3 = incidência de degenerescência moderada com 11 a $30 \%$ da polpa afetada; e $4=$ incidência de degenerescência severa, com mais de $30 \%$ da polpa afetada.

Para as determinações dos teores de $\mathrm{Ca}$, Mg e K (mg.kg-1 de massa fresca), foram utilizadas metodologias propostas por Tedesco et al. (1995), em que se retirou uma fatia longitudinal (cunha) com 1 $\mathrm{mm}$ de espessura da polpa dos frutos de cada escala de severidade. Estas amostras foram trituradas e homogeneizadas com um multiprocessador RI $6720 \mathrm{e}$ um mixer Braun Multiquick MR40, respectivamente. Em seguida, foram pesados cerca de 5,0 gramas de polpa em uma balança analítica, depositados em cadinhos de porcelana $\mathrm{M}-2$ e incinerados em mufla, a uma temperatura de $600{ }^{\circ} \mathrm{C}$, por quatro horas. Após o resfriamento, as cinzas de cada amostra foram solubilizadas por meio da adição de $16 \mathrm{~mL}$ de $\mathrm{HCl}$ $1,8 \mathrm{M}$.

Para a determinação de $\mathrm{Ca}$, retirou-se uma alíquota de $5 \mathrm{~mL}$ do extrato original e adicionaram-se $5 \mathrm{ml}$ de lantânio no interior de um tubo Falcon de 15 $\mathrm{mL}$, para então efetuar-se a leitura em um espectrofotômetro de absorção atômica (AA), Perkin - Elmer modelo A-analyst 100.

Para a determinação de $\mathrm{Mg}$, retiraram-se 2 $\mathrm{mL}$ do extrato original sobre os quais se adicionaram $10 \mathrm{~mL}$ de água destilada. Desta solução diluída $(2 \mathrm{~mL}$ de extrato $+10 \mathrm{~mL}$ de água destilada), pipetaram-se $3 \mathrm{~mL}$ da solução e adicionaram-se $3 \mathrm{~mL}$ de lantânio. Em seguida, efetuou-se a leitura no mesmo aparelho utilizado para a determinação de $\mathrm{Ca}$.

Para a quantificação de $\mathrm{K}$, retirou-se uma alíquota de $3 \mathrm{~mL}$ do extrato original, seguidas de adição de $20 \mathrm{~mL}$ de água destilada, e homogeneização. A quantificação foi efetuada em um fotômetro de chama Digimed DM-61.

Os dados foram submetidos à análise da variância (ANOVA), com o auxílio do programa SAS (SAS Institute, 2002). Dados em porcentagem foram transformados pela fórmula arco-seno $[(\mathrm{x}+0,5) / 100]^{1 / 2}$ antes de serem submetidos à ANOVA. Para a comparação das médias, adotou-se o teste de Tukey $(\mathrm{p}<0,05)$. 


\section{RESULTADOS E DISCUSSÃO}

As variáveis analisadas no momento da colheita não foram afetadas pelos porta-enxertos, com exceção da AT, na qual os frutos oriundos de árvores com o porta-enxerto Marubakaido com filtro M9 apresentaram os maiores valores (dados não apresentados).

A taxa de produção de etileno foi menor nos frutos armazenados na condição de $2 \mathrm{kPa}$ de $\mathrm{CO}_{2}$ do que os frutos armazenados na condição de $0,5 \mathrm{kPa}$ de $\mathrm{CO}_{2}$, tanto no momento da saída da câmara quanto após sete dias de exposição em condição ambiente (Tabela 1). Os efeitos positivos de condições de armazenamento com concentrações elevadas de $\mathrm{CO}_{2}$ devem estar relacionadas ao efeito inibitório do $\mathrm{CO}_{2}$ na ação do etileno, reduzindo assim sua produção autocatalítica (BLANKENSHIP; DOLE, 2003).

Os frutos armazenados na condição de 1,2 $\mathrm{kPa}+2 \mathrm{kPa}$ de $\mathrm{CO}_{2}$ foram os que apresentaram a coloração da epiderme mais verde (maior $h^{\circ}$ ) (Tabela 1). Em outros trabalhos, tem sido verificado que a elevação do $\mathrm{CO}_{2}$ no armazenamento causa uma retenção na cor de fundo verde da epiderme em maçãs (BRACKMANN et al., 2005; CORRÊA et al., 2010). Entre porta-enxertos, a maior retenção da cor verde da epiderme foi observada nos frutos provenientes das árvores com o MM-106, que é caracterizado por ser um porta-enxerto menos vigoroso. Remorini et al. (2008), ao compararem a qualidade de frutos em diferentes porta-enxertos em pêssegos, também encontraram melhor manutenção de cor verde da epiderme nos frutos provenientes do porta-enxerto menos vigoroso, concordando com os dados do presente trabalho. Porta-enxertos menos vigorosos favorecem maior penetração de luz no dossel da planta, o que pode acarretar maior acúmulo de clorofilas na epiderme, resultando em maior retenção da cor verde nos frutos (GIORGI et al., 2005).

A condição de armazenamento de $2 \mathrm{kPa}$ de $\mathrm{CO}_{2}$ apresentou frutos com melhor resistência para a força para a penetração da polpa (Tabela 1). Isto se deve ao fato de que condições de armazenamento em que há combinações de baixo $\mathrm{O}_{2}$ e alto $\mathrm{CO}_{2}$ resultam em menor atividade de enzimas hidrolíticas de parede celular (BRACKMANN et al., 2002b). Os frutos oriundos de árvores com porta-enxertos MM-106 e Marubakaido com filtro M-9 apresentaram maiores valores de força para penetração da polpa do que os frutos com porta-enxerto Marubakaido. Rato et al. (2008), ao realizarem trabalho com ameixas oriundas de árvores com diferentes porta-enxertos, também encontraram diferenças, porém associaram a maior manutenção deste atributo aos maiores teores de Ca nestes frutos, pois este nutriente está envolvido na manutenção da integridade da parede celular. Esta afirmação concorda em parte com os resultados obtidos no presente trabalho, em que os frutos provenientes do porta-enxerto MM-106, além de apresentarem maior força para a penetração da polpa, também apresentaram maior teor de Ca (Tabela $3)$.

A AT foi maior nos frutos oriundos de árvores com o porta-enxerto Marubakaido com filtro M-9 em relação aos demais, em ambas as condições de armazenamento (Tabela 2). Isto se deve ao fato de que, no momento da colheita, os frutos deste portaenxerto já apresentavam maior AT quando comparados aos frutos dos demais porta-enxertos (dados não apresentados).

Os maiores teores de SS foram encontrados nos frutos armazenados na condição de $1,2 \mathrm{kPa} \mathrm{O}_{2}+$ $0,5 \mathrm{kPa}$ de $\mathrm{CO}_{2}$ (Tabela 2). Isto provavelmente está relacionado ao maior conteúdo de pectinas solúveis, uma vez que esses frutos apresentaram a menor força para penetração da polpa. Relação inversa entre valores de consistência da polpa e SS também foi observada em goiabas 'Pedro Sato' (STEFFENS et al., 2008) e ameixas 'Laetitia' (STEFFENS et al., 2009) armazenadas sob refrigeração. Entre os portaenxertos, o Marubakaido com filtro M-9 apresentou os frutos com os maiores valores de SS. A diferença no teor de SS entre porta-enxertos também foi verificada em pêssegos por Mathias et al. (2008). Porém, estes autores afirmam que isto ocorre em plantas que estão mais expostas a raios solares, proporcionados pelo formato da copa, causando aumento da taxa fotossintética e, consequentemente, maior acúmulo de açúcares nos frutos. Todavia, este efeito também deveria ter sido observado em frutos de plantas sob MM-106.

$\mathrm{Na}$ condição de baixo $\mathrm{CO}_{2}\left(<0,5\right.$ de $\left.\mathrm{CO}_{2}\right)$, não houve diferença quanto à incidência e severidade de degenerescência de polpa entre os porta-enxertos (Tabela 2). Porém, na condição de $2 \mathrm{kPa}$ de $\mathrm{CO}_{2}$ plantas com os porta-enxertos Marubakaido e $\mathrm{Ma}^{2}$ rubakaido com filtro M-9 apresentaram frutos com maior incidência do distúrbio, se comparados aos frutos armazenados em baixo $\mathrm{CO}_{2}\left(<0,5 \mathrm{de} \mathrm{CO}_{2}\right)$, sendo que aqueles oriundos de árvores com o porta-enxerto Marubakaido também apresentaram maior severidade (Tabela 2). Em outros trabalhos, tem sido verificado que o alto $\mathrm{CO}_{2}$ no armazenamento em $\mathrm{AC}$ contribui para maior degenerescência de polpa em maçãs 'Fuji'(BRACKMANN et al., 1999; CORRÊA et al., 2010), pois estas condições favorecem a mudança da respiração aeróbica para a respiração anaeróbica dos frutos, resultando assim no acúmulo de substâncias voláteis e induzindo a manifestação de distúrbios fisiológicos. Quanto aos porta-enxertos, inexistem trabalhos no Brasil, mostrando o efeito destes sobre a ocorrência da degenerescência de polpa em maçãs, nem tampouco estudando os mecanismos de como isto ocorre. Porém, os dados de composição mineral de frutos (Tabela 3 ) mostram que o tipo de porta-enxerto afeta os teores de nutrientes nos frutos.

Os teores de nutrientes na polpa de frutos 
diferiram entre os porta-enxertos, sendo que os frutos oriundos de árvores com o MM-106 foram os que apresentaram os mais elevados teores de $\mathrm{Ca}$, os menores de $\mathrm{K}$ e as maiores relações $\mathrm{K} / \mathrm{Ca}$, $\mathrm{Mg} / \mathrm{Ca}$ e $\mathrm{K}+\mathrm{Mg} / \mathrm{Ca}$ (Tabela 3). Rato et al. (2008) encontraram diferenças na concentração de $\mathrm{Ca} \mathrm{em}$ ameixas provenientes de diferentes porta-enxertos. Para Martínez-Ballesta et al. (2010), os teores de Ca e Mg nas plantas de maneira geral são fortemente relacionados ao porta-enxerto, pois as características fisiológicas dos mesmos afetam a absorção e a translocação destes minerais para a planta.

Os porta-enxertos Marubakaido e Marubakaido com filtro M-9 apresentaram frutos com menores teores de $\mathrm{Ca}$, maiores teores $\mathrm{K}$ e as maiores relações $\mathrm{K} / \mathrm{Ca}$ (Tabela 3), além de apresentarem maior incidência e severidade de degenerescência de polpa (Tabela 2). Estes resultados concordam com os obtidos por Andziak e Tomala (2004), que observaram elevada incidência de distúrbios fisiológicos no armazenamento em maçãs com baixo teor de $\mathrm{Ca}$ e alto de K. O Ca é um importante nutriente, sendo sua deficiência relacionada com o aparecimento de distúrbios fisiológicos em frutos (ANDZIAK; TOMALA, 2004; JAMES; JOBLIN, 2009; FREITAS et al., 2010; AMARANTE et al., 2010). Em frutos armazenados por períodos prolongados, ocorre a degradação da lamela média, sendo mais intensa em frutos com deficiência de $\mathrm{Ca}$, sendo que este elemento também controla a estabilidade celular e, consequentemente, a membrana plasmática. No entanto, estes aspectos ainda precisam ser mais bem estudados (JAMES; JOBLING, 2009). O K é um nutriente antagonista ao Ca (NEUWALD et al., 2008), cujo efeito é devido a uma competição com o Ca por sítios de ligação na membrana plasmática (JAMES; JOBLING, 2009).

De maneira geral, percebe-se que pode existir uma relação entre teores de $\mathrm{Ca}$ e $\mathrm{K}$ nos frutos e as relações $\mathrm{K} / \mathrm{Ca}, \mathrm{Mg} / \mathrm{Ca}$ e $(\mathrm{K}+\mathrm{Mg}) / \mathrm{Ca}$ com a incidência da degenerescência de polpa em maçãs 'Fuji' após o armazenamento em AC.

TABELA 1-Taxa de produção de etileno na saída da câmara e após sete dias de exposição em condição ambiente, cor da epiderme (ângulo 'hue', $h^{\circ}$ ) e força para a penetração da polpa de maçãs 'Fuji' oriundas de árvores com três porta-enxertos, após serem armazenadas por oito meses, em duas condições de atmosfera controlada, seguidos de sete dias de exposição em condições ambiente.

\begin{tabular}{lllll}
\hline & \multicolumn{3}{c}{ Porta-enxerto } \\
\hline $\mathrm{O}_{2}+\mathrm{CO}_{2}(\mathrm{kPa})$ & $\mathrm{MM}-106$ & Marubakaido & Marubakaido com filtro M-9 & Média \\
\hline
\end{tabular}

Taxa de produção de etileno $\left(\mathrm{pmol} \mathrm{kg}^{-1} \mathrm{~s}^{-1}\right)$

\begin{tabular}{|c|c|c|c|c|}
\hline & \multicolumn{4}{|c|}{ Saída da câmara } \\
\hline $1,2+<0,5$ & 0,14 & 0,21 & 0,19 & $0,18 \mathrm{a}$ \\
\hline $1,2+2,0$ & 0,04 & 0,03 & 0,08 & $0,05 \mathrm{~b}$ \\
\hline Média & $0,09 \mathrm{~A}$ & $0,12 \mathrm{~A}$ & $0,13 \mathrm{~A}$ & \\
\hline CV (\%) & \multicolumn{4}{|c|}{63,61} \\
\hline & \multicolumn{4}{|c|}{ Após sete dias } \\
\hline $1,2+<0,5$ & 0,49 & 0,27 & 0,21 & $0,32 \mathrm{a}$ \\
\hline $1,2+2,0$ & 0,01 & 0,02 & 0,02 & $0,02 b$ \\
\hline Média & $0,25 \mathrm{~A}$ & $0,14 \mathrm{~A}$ & $0,11 \mathrm{~A}$ & \\
\hline CV $(\%)$ & \multicolumn{4}{|c|}{91,08} \\
\hline
\end{tabular}

\begin{tabular}{lcccc}
\hline \multicolumn{5}{c}{ Cor da epiderme $\left({ }^{\circ} h\right)$} \\
\hline $1,2+<0,5$ & 101,31 & 99,85 & 99,93 & $100,36 \mathrm{~b}$ \\
$1,2+2,0$ & 104,63 & 101,51 & 101,46 & $102,53 \mathrm{a}$ \\
\hline Média & $102,97 \mathrm{~A}$ & $100,68 \mathrm{~B}$ & $100,70 \mathrm{~B}$ \\
$\mathrm{CV}(\%)$ & & \multicolumn{2}{c}{1,05} \\
\hline
\end{tabular}

Força para a penetração da polpa $(\mathrm{N})$

\begin{tabular}{lcccc}
\hline $1,2+<0,5$ & 2,67 & 2,51 & 2,67 & $2,61 \mathrm{~b}$ \\
$1,2+2,0$ & 2,92 & 2,72 & 2,83 & $2,83 \mathrm{a}$ \\
\hline Média & $2,79 \mathrm{~A}$ & $2,62 \mathrm{~B}$ & \multicolumn{2}{c}{$2,75 \mathrm{~A}$} \\
CV $(\%)$ & & \multicolumn{2}{c}{3,09} & \\
\hline
\end{tabular}

Médias seguidas pela mesma letra, maiúscula nas linhas e minúscula nas colunas, não diferem entre si, pelo teste de Tukey ( $<<0,05)$. 
TABELA 2 -Acidez titulável, sólidos solúveis e incidência e severidade de degenerescência da polpa de maçãs 'Fuji' oriundas de árvores com três porta-enxertos, após serem armazenadas por oito meses, em duas condições de atmosfera controlada, seguidos de sete dias de exposição em condições ambiente.

\begin{tabular}{|c|c|c|c|c|}
\hline \multirow[b]{2}{*}{$\mathrm{O}_{2}+\mathrm{CO}_{2}(\mathrm{kPa})$} & \multicolumn{4}{|c|}{ Porta-enxerto } \\
\hline & MM-106 & Marubakaido & Marubakaido com filtro M-9 & Média \\
\hline & \multicolumn{4}{|c|}{ Acidez titulável (meq $\left.100 \mathrm{~mL}^{-1}\right)$} \\
\hline $1,2+<0,5$ & $3,12 \mathrm{Ba}$ & $4,33 \mathrm{Ba}$ & $5,85 \mathrm{Aa}$ & - \\
\hline $1,2+2,0$ & $3,28 \mathrm{Ba}$ & $3,68 \mathrm{Ba}$ & $5,51 \mathrm{Aa}$ & - \\
\hline Média & - & - & - & \\
\hline \multirow[t]{2}{*}{$\mathrm{CV}(\%)$} & \multicolumn{4}{|c|}{13,48} \\
\hline & \multicolumn{4}{|c|}{ Sólidos solúveis ( ${ }^{\circ}$ Brix) } \\
\hline $1,2+<0,5$ & 13,95 & 13,50 & 14,65 & $14,03 \mathrm{a}$ \\
\hline $1,2+2,0$ & 13,05 & 13,00 & 14,20 & $13,42 b$ \\
\hline Média & $13,50 \mathrm{~B}$ & $13,25 \mathrm{~B}$ & $14,42 \mathrm{~A}$ & \\
\hline \multirow[t]{2}{*}{ CV $(\%)$} & \multirow{2}{*}{\multicolumn{4}{|c|}{$\frac{4,77}{\text { Incidência de degenerescência da polpa (\%) }}$}} \\
\hline & & & & \\
\hline $1,2+<0,5$ & $1,25 \mathrm{Aa}$ & $1,68 \mathrm{Ab}$ & $1,27 \mathrm{Ab}$ & - \\
\hline $1,2+2,0$ & $3,16 \mathrm{Ba}$ & $7,82 \mathrm{Aa}$ & $18,65 \mathrm{Aa}$ & - \\
\hline Média & - & - & - & \\
\hline \multirow{2}{*}{$\mathrm{CV}(\%)$} & \multicolumn{4}{|c|}{30,57} \\
\hline & \multicolumn{4}{|c|}{ Severidade de degenerescência da polpa (1-4) } \\
\hline $1,2+<0,5$ & $1,01 \mathrm{Aa}$ & $1,14 \mathrm{Aa}$ & $1,02 \mathrm{Ab}$ & - \\
\hline $1,2+2,0$ & $1,06 \mathrm{Ba}$ & $1,12 \mathrm{Ba}$ & $1,33 \mathrm{Aa}$ & - \\
\hline Média & - & - & - & \\
\hline CV $(\%)$ & \multicolumn{4}{|c|}{6,86} \\
\hline
\end{tabular}

Médias seguidas pela mesma letra, maiúscula nas linhas e minúscula nas colunas, não diferem entre si, pelo teste de Tukey $(\mathrm{p}<0,05)$

TABELA 3 - Teor de nutrientes totais na polpa de maçãs 'Fuji’ oriundas de árvores com três porta-enxertos, após serem armazenadas por oito meses, em duas condições de atmosfera controlada, seguidos de sete dias de exposição em condições ambiente.

\begin{tabular}{lcccccccc}
\hline \multirow{2}{*}{ Porta-enxerto } & \multicolumn{3}{c}{ Teores minerais $\left(\mathrm{mg} \mathrm{kg}^{-1}\right)$} & & \multicolumn{3}{c}{ Relações minerais } \\
\cline { 2 - 3 } & $\mathrm{Ca}$ & $\mathrm{Mg}$ & $\mathrm{K}$ & & $\mathrm{K} / \mathrm{Ca}$ & $\mathrm{Mg} / \mathrm{Ca}$ & $(\mathrm{K}+\mathrm{Mg}) / \mathrm{Ca}$ \\
\hline MM-106 & $87 \mathrm{a}$ & $48 \mathrm{a}$ & $632 \mathrm{~b}$ & & $7,4 \mathrm{~b}$ & & $0,6 \mathrm{~b}$ & $632,4 \mathrm{~b}$ \\
Marubakaido & $72 \mathrm{~b}$ & $47 \mathrm{a}$ & $760 \mathrm{a}$ & & $10,8 \mathrm{a}$ & $0,7 \mathrm{a}$ & $761,2 \mathrm{a}$ \\
Marubakaido c/ filtro M-9 & $75 \mathrm{~b}$ & $51 \mathrm{a}$ & $709,5 \mathrm{a}$ & & $9,5 \mathrm{a}$ & $0,7 \mathrm{a}$ & $710,2 \mathrm{a}$ \\
\hline $\mathrm{CV}(\%)$ & 12,22 & 12,57 & 14,21 & & 15,86 & & 12,57 & 14,20 \\
\hline
\end{tabular}

Médias seguidas pela mesma letra na vertical não diferem entre si, pelo teste de Tukey $(\mathrm{p}<0,05)$.

\section{CONCLUSÕES}

1-O tipo de porta-enxerto utilizado tem pouca influência na qualidade de maçãs 'Fuji' armazenadas em atmosfera controlada.

2-A condição de atmosfera controlada utilizada, com alto $\mathrm{CO}_{2}(2 \mathrm{kPa})$, retarda o amadurecimento de maçãs 'Fuji', contudo contribui para maior incidência e severidade de degenerescência de polpa.
3-A degenerescência de polpa em maçãs 'Fuji' está relacionada com o tipo de porta-enxerto, sendo o MM-106 o mais recomendado para frutos a serem armazenados em atmosfera controlada, pois é o que apresenta menos predisposição dos frutos a distúrbio fisiológico e proporciona frutos com maiores teores de cálcio e menores relações $\mathrm{K} / \mathrm{Ca}$, $\mathrm{Mg} / \mathrm{Ca}$ e $(\mathrm{K}+\mathrm{Mg}) / \mathrm{Ca}$. 


\section{AGRADECIMENTOS}

Ao Conselhlógico (CNPq), pelo apoio financeiro a este projeto.

\section{REFERÊNCIAS}

AMARANTE, C.V.T. do; STEFFENS, C.A.; ERNANI, P.R. Identificação pré-colheita do risco de ocorrência de "bitter pit" em maçãs 'Gala' por meio de infiltração com magnésio e análise dos teores de cálcio e nitrogênio nos frutos. Revista Brasileira de Fruticultura, Jaboticabal, v. 32, p.27-34, 2010.

ANDZIAK, J.; TOMALA, K. Influence of rootstocks on mineral nutrition, fruit maturity and quality of 'Jonagold' apples. Sodininkyste ir Darzininkyste, Lithuanian Babtai, v.23, n.1, p.20-32, 2004.

BLANKENSHIP, S.M.; DOLE, J.M. 1-Methylcyclopropene: a review. Postharvest Biology and Technology, Amsterdam, v.28, p.1-25, 2003.

BRACKMANN, A.; BENEDETTI, M.; STEFFENS, C. A.; MELLO, A. M. de. Efeito da temperatura e condições de atmosfera controlada na armazenagem de maçãs 'Fuji' com incidência de pingo de mel. Revista Brasileira de Agrociência, Pelotas, v. 8, n. 1, p.37-45, 2002a.

BRACKMANN, A.; BORTOLUZZI, G.; BORTOLUZ, L. Controle da degenerescência da polpa da maçã 'Fuji' com concentrações dinâmicas de $\mathrm{O}_{2} \mathrm{e}$ $\mathrm{CO}_{2}$ e redução da umidade relativa durante o armazenamento em atmosfera controlada. Ciência Rural, Santa Maria, v.29, p.459-463, 1999.

BRACKMANN, A.; GUARIENTI, A.J.W.; SAQUET, A.A.; GIEHL, R.F.H.; SESTARI, I. Condições de atmosfera controlada para maçã 'Pink Lady'. Ciência Rural, Santa Maria, v.35, p.504-509, 2005.

BRACKMANN, A.; STEFFENS, C.A.; WACLAWOSKY, A.J. Influência da época de colheita e do armazenamento em atmosfera controlada na qualidade da maçã 'Braeburn'. Pesquisa Agropecuária Brasileira, Brasília,v.37, p.295-301, 2002 b.

CANTILLANO, F.F.; GIRARDI, C.L. Distúrbios fisiológicos. In: EMBRAPA. (Org.). Frutas do Brasil, Maçã Pós-colheita. Brasília: EMBRAPA, 2004, v.39, p.45-53.
CORRÊA, T.R.; STEFFENS, C.A.; AMARANTE, C.V.T.; BRACKMANN, A.; SILVEIRA, J.P.G.; TANAKA, H.; BOTH, V. Qualidade de maçãs 'Fuji' armazenadas em atmosfera controlada e influência do clima na degenerescência da polpa. Pesquisa Agropecuária Brasileira, Brasília, v.45, n.6, p.531538,2010 .

FREITAS, S.T. DE; AMARANTE, C.V.T. DO; LABAVITCH, J.M.; MITCHAM, E. Cellular approach to understand bitter pit development in apple fruit. Postharvest Biology and Technology, Amsterdam, v. 57, p. 5-13, 2010.

GIORGI, M.; CAPOCASA, F.; SCALZO, J.; MURRI, G.; BATTINO, M.; MEZZETTI, B. The rootstock effects on plant adaptability, production, fruit quality, and nutrition in the peach (cv. 'Suncrest'). Scientia Horticulturae, Amsterdam, v.107, p.36-42, 2005.

IUCHI, V. L.; IUCHI, T. Descrição dos distúrbios fisiológicos da macieira. In: , EPAGRI. Distúrbios fisiológicos nutricionais em macieira. Florianópolis, 2001. p.12-61.

JAMES, H.J.; JOBLING, J.J. Contrasting the structure and morphology of the radial and diffuse flesh browning disorders and $\mathrm{CO}_{2}$ injury of 'Cripps Pink' apples. Postharvest Biology and Technology, Amsterdam, v.53, p.36-42, 2009.

MARTÍNEZ-BALLESTA, C.M.; ALCARAZLÓPEZ, C.; MURIES, B.; MOTA-CADENAS,C.; CARJAL, M.Physiological aspects of rootstockscion interactions. Scientia Horticulturae, Amsterdam, v.127, p.112-118, 2010.

MATHIAS, C.; MAYER, N.A.;MATTIUZ,B.H.; PEREIRA, F.M. Efeito de porta-enxertos e espaçamentos entre plantas na qualidade de pêssegos 'Aurora'. Revista Brasileira de Fruticultura, Jaboticabal, v.30, n.1, p.165-170, 2008.

NEUWALD, D.A.; KITTEMANN, D.; STREIF, J. Possible prediction of physiological storage disorders in 'Braeburn' apples comparing fruit of different orchards. Acta Horticulturae, Wellington, n. 796, p. 211-216, 2008.

RATO, A.E.; AGULHEIRO, A.C.; BARROSO, J.M.; RIQUELME, A.F. Soil and rootstock influence on fruit quality of plums (Prunus domestica L.). Scientia Horticulturae, Amsterdam, v.118 p 218-222, 2008. 
REMORINI, D.; TAVARINI, S.; DEGL'INNOCENTI, E.; LORETI, F.; MASSAI, R.; GUIDI, L. Effect of rootstocks and harvesting time on the nutritional quality of peel and flesh of peach fruits. Food Chemistry, Amsterdam, v.110, p. 361-367, 2008.

SAS INSTITUTE. Getting started with the SAS learning edition. Cary: SAS Institute, 2002. 200p.

SAURE, M. C. Calcium translocation to fleshy fruit: its mechanism and endogenous control. Scientia Horticulturae, Amsterdam, n.105, p.65-89, 2005.
STEFFENS, C.A.; AMARANTE, C.V.T.; ALVES, E.O.; TANAKA, H.; BRACKMANN, A.; BOTH, V. Armazenamento de ameixas 'Laetitia' em atmosfera modificada. Ciência Rural, Santa Maria, v.39, n.9, p.2439-2444, 2009.

STEFFENS, C.A.; AMARANTE, C.V.T. DO; SILVEIRA, J.P.G. DA; CHECHI, R.; ESPÍNDOLA, B.P. Tolerância ao dano pelo frio em goiabas 'Pedro Sato' submetidas ao condicionamento térmico. Biotemas, Florianópolis, v.21, n.3, p.75-80, 2008.

TEDESCO, M.J.; GIANELLO, C.; BISSANI, C.A.; BOHNEN, H.; VOLKWEISS, S.J. Análise do solo, plantas e outros materiais. 2. ed. Porto Alegre: Departamento de Solos, UFRGS, 1995. 174p. (Boletim Técnico de Solos, 5). 